\title{
A GENERAL DIFFERENTIAL EQUATION FOR CLASSICAL POLYNOMIALS
}

B. D. AGRAWAL AND I. K. KHANNA

1. Introduction. The object of the present paper is to derive the two differential equations satisfied by the polynomial set $B_{n}(x, y)$ and the general solutions thereof. $B_{n}(x, y)$, the generalization of as many as eighteen classical polynomials such as Laguerre polynomials, Hermite polynomials, Legendre polynomials, Jacobi polynomials, Bedient polynomials etc., has been defined by us by means of the generating relation

$$
\sum_{n=0}^{\infty} B_{n}(x, y) t^{n}={ }_{p} F_{q}\left[\left(\alpha_{p}\right) ;\left(\beta_{q}\right) ; \nu x t\right]_{r} F_{s}\left[\left(a_{r}\right) ;\left(b_{s}\right) ; \mu y^{-m t^{m}}\right],
$$

valid under the conditions given in [1]. Several other results for the polynomial set $B_{n}(x, y)$ have also been given in [1].

The following notations have been used for brevity:

(i) $(m) \equiv 1,2, \cdots, m$.

(ii) $\left(a_{p}\right) \equiv a_{1}, a_{2}, \cdots, a_{p}$.

(iii) $\left(a_{p ; s}\right) \equiv a_{1}, a_{2}, \cdots, a_{s-1}, a_{s+1}, \cdots, a_{p}$.

(iv) $\left[\left(a_{p}\right)\right]_{n} \equiv\left(a_{1}\right)_{n}\left(a_{2}\right)_{n} \cdots\left(a_{p}\right)_{n}$.

(v) $\Delta(a ; b) \equiv b / a,(b+1) / a, \cdots,(b+a-1) / a$.

(vi) $\Delta_{k}[a ; b] \equiv(b / a)_{k}((b+1) / a)_{k} \cdots((b+a-1) / a)_{k}$.

(vii) $\Delta\left(m ; 1+\left(b_{q}\right)\right) \equiv\left(\left(r+b_{j}\right) / m\right), j=1, q ; r=1, m$.

(viii) $\Delta_{k}\left[m ; 1+\left(b_{q}\right)\right] \equiv \prod_{j=1}^{g} \prod_{r=1}^{m}\left(\left(r+b_{j}\right) / m\right)_{k}$.

(ix) $\nabla\left(m(g) ; 1-g+\alpha_{h}-\left(\alpha_{p ; n}\right)+m\right)$ stands for the parameters obtained from the sequence

$$
\left(\frac{i-g+\alpha_{h}-\alpha_{j}+m}{m}\right) \quad i=1, m ; j=1, p
$$

after deleting the particular parameter 1 from it, the latter being obtained by putting $i=g$ and $j=h$ simultaneously in the above sequence.

2. Differential equations for $B_{n}(x, y)$. (i) Let us take

$$
W_{1}={ }_{m q+r+r} F_{m p+s}\left(\begin{array}{l}
\Delta(m ;-n), \Delta\left(m ; 1-\left(\beta_{q}\right)-n\right),\left(a_{r}\right) ; G x^{-m} \\
\Delta\left(m ; 1-\left(\alpha_{p}\right)-n\right),\left(b_{\varepsilon}\right)
\end{array}\right) .
$$

Received by the editors December 24, 1968. 
Since $\theta x^{-m}=-m x^{-m}$, where $\theta \equiv x \partial / \partial x$, it follows that $\left\{\theta\left[\left(b_{s}\right)-\theta / m-1\right] \Delta\left[m ; 1-\left(\alpha_{p}\right)-n-m-\theta\right]\right\} W_{1}$

$=\sum_{k=1}^{[n / m]} \frac{\left((-m k) G^{k} \Delta_{k}[m ;-n] \Delta_{k}\left[m ; 1-\left(\beta_{q}\right)-n\right]\left[\left(a_{r}\right)\right]_{k}\left[k+\left(b_{s}\right)-1\right] \Delta\left[m ; 1-\left(\alpha_{p}\right)-n+m k-m\right]\right)}{k !\left[\left(b_{s}\right)\right]_{k} \Delta_{k}\left[m ; 1-\left(\alpha_{p}\right)-n\right]_{k}}$ $=-m \sum_{k=1}^{[n / m]} \frac{\Delta_{k}[m ;-n] \Delta_{k}\left[m ; 1-\left(\beta_{q}\right)-n\right]\left[\left(a_{r}\right)\right]_{k} G^{k} x^{-m k}}{(k-1) !\left[\left(b_{s}\right)\right]_{k-1} \Delta_{k-1}\left[m ; 1-\left(\alpha_{p}\right)-n\right]}$

$=-m \sum_{k=0}^{[(n-m) / m]} \frac{\Delta_{k+1}[m ;-n] \Delta_{k+1}\left[m ; 1-\left(\beta_{q}\right)-n\right]\left[\left(a_{r}\right)\right]_{k+1} G^{k+1} x^{-m(k+1)}}{k !\left[\left(b_{s}\right)\right]_{k} \Delta_{k}\left[m ; 1-\left(\alpha_{p}\right)-n\right]}$

$=-m G \sum_{k=0}^{[n / m]} \frac{\Delta_{k}[m ;-n] \Delta_{k}\left[m ; 1-\left(\beta_{q}\right)-n\right]\left[\left(a_{r}\right)\right]_{k} G^{k} x^{-m k-m}}{k !\left[\left(b_{s}\right)\right]_{k} \Delta_{k}\left[m ; 1-\left(\alpha_{p}\right)-n\right]}$

$\times \Delta[m ;-n+k m] \Delta\left[m ; 1-\left(\beta_{q}\right)-n+m k\right]\left[\left(a_{r}\right)+k\right]$,

for $\Delta[m ;-n+k m]=0$ in the interval $[(n-m+1) / m] \leqq k \leqq[n / m]$. Hence

$$
\begin{aligned}
& \left\{\theta\left[\left(b_{s}\right)-\theta / m-1\right] \Delta\left[m ; 1-\left(\alpha_{p}\right)-n-m-\theta\right]\right. \\
& \left.+m G x^{-m} \Delta[m ;-n-\theta]\left[\left(a_{r}\right)-\theta / m\right] \times \Delta\left[m ; 1-\left(\beta_{q}\right)-n-\theta\right]\right\} W_{1}=0,
\end{aligned}
$$
i.e.

$$
\begin{gathered}
\left\{\theta\left[\theta-m\left(b_{s}\right)+m\right]\left[\theta-(m)+\left(\alpha_{p}\right)+n+m\right]-G(-m)^{m(p-q-1)+s-r+1}\right. \\
\left.\times x^{-m}[\theta+n+1-(m)]\left[\theta-(m)+\left(\beta_{q}\right)+n\right]\left[\theta-m\left(a_{r}\right)\right]\right\} W_{1}=0 .
\end{gathered}
$$

Now, let $W_{1}=(1 / H) x^{-n} W$. Hence $\theta\left\{W_{1}\right\}=(1 / H) x^{-n}(\theta-n) W$. Therefore, (2.2) becomes

$$
\begin{aligned}
& (1 / H) x^{-n}\left\{(\theta-n)\left[\theta-n-m\left(b_{s}\right)+m\right]\left[\theta-(m)+\left(\alpha_{p}\right)+m\right]\right. \\
& \quad-G(-m)^{m(p-q-1)+8-r+1} \\
& \left.\quad \times x^{-m}[\theta-(m)+1]\left[\theta-(m)+\left(\beta_{q}\right)\right]\left[\theta-n-m\left(a_{r}\right)\right]\right\} W=0 .
\end{aligned}
$$

Now

$$
G=\frac{\mu(-m)^{m(q-p+1)}}{(\nu y)^{m}} \text { and } H=\frac{\left[\left(\alpha_{p}\right)\right]_{n} \nu^{n}}{n !\left[\left(\beta_{q}\right)\right]_{n}}
$$

makes $W=B_{n}(x, y)$ and (2.3) transforms itself into

$$
\begin{aligned}
\{(\theta-n)[\theta- & \left.n-m\left(b_{s}\right)+m\right]\left[\theta-(m)+\left(\alpha_{p}\right)+m\right] \\
& -\frac{(-m)^{--r+1} \mu}{(\nu x y)^{m}}[\theta-(m)+1] \\
& \left.\times\left[\theta-(m)+\left(\beta_{q}\right)\right]\left[\theta-n-m\left(a_{r}\right)\right]\right\} B_{n}(x, y)=0,
\end{aligned}
$$


which is one of the differential equations for the polynomial set $B_{n}(x, y)$.

(ii) Similarly, it can also be shown that the partial differential equation for $B_{n}(x, y)$ in $\phi$, where $\phi \equiv y \partial / \partial y$, is given by

$$
\begin{aligned}
\left\{\phi \left[\phi-m\left(b_{\varepsilon}\right)\right.\right. & +m]\left[\phi+\left(\alpha_{p}\right)-(m)+n+m\right] \\
& -\frac{\mu(-m)^{s-r+1}}{(\nu x y)^{m}}[\phi+n-(m)+1] \\
& \left.\times\left[\phi+\left(\beta_{q}\right)+n-(m)\right]\left[\phi-m\left(a_{r}\right)\right]\right\} B_{n}(x, y)=0 .
\end{aligned}
$$

3. Other solutions of the differential equations. (i) The complete solution of the differential equation (2.4) is given by

$$
W=A_{0} W_{0}+\sum_{t=1}^{s} A_{t} W_{t}+\sum_{o=1}^{m} \sum_{h=1}^{p} A_{o, h} W_{o, h},
$$

where $A_{0}, A_{t}, A_{0, h}$ are arbitrary constants and

$$
W_{0}=B_{n}(x, y) \text {, }
$$

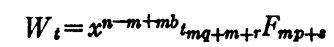

$$
\cdot\left(\begin{array}{rrr}
\Delta\left(m ;-n+m-m b_{t}\right),\left(\left(a_{r}\right)-b_{t}+1\right), \Delta\left(m ;-n+m-m b_{t}-\left(\beta_{q}\right)+1\right) & \\
\Delta\left(m ;-n+m-m b_{t}-\left(\alpha_{p}\right)+1\right), & \left(\left(b_{s_{i}}\right)-b_{t}+1\right), & \left(2-b_{t}\right)
\end{array}\right.
$$

and

$W_{0, h}=x^{\sigma-m-\alpha_{h_{m q+m+r}}} F_{m p+\sigma}$

$$
\left[\begin{array}{rr}
\Delta\left(m ; \alpha_{h}+m-g\right), \Delta\left(m ; 1-g-\left(\beta_{q}\right)+\alpha_{h}+m\right),\left(\left(a_{r}\right)+1+\frac{\alpha_{h}+n-g}{m}\right) & \\
\nabla\left(m(g) ; 1-g+\alpha_{h}-\left(\alpha_{p} ; h\right)+m\right),\left(2+\frac{n-g+\alpha_{h}}{m}\right),\left(\left(b_{s}\right)+1+\frac{n-g+\alpha_{h}}{m}\right)
\end{array} \mid .\right.
$$

Note. It is worth noting that the $s+1$ solutions $W_{0}$ and $W_{t}$ of the differential equation (2.4) are polynomials, whereas $W_{o, h}$ are $m p$ functions.

(ii) Proceeding as above, it can also be shown that the complete solution of the partial differential equation (2.5) is given by

$$
W=A_{0}^{\prime} W_{0}+\sum_{t=1}^{s} A_{t}^{\prime} W_{t}^{\prime}+\sum_{g=1}^{m} \sum_{h=1}^{p} C_{g, h}^{\prime} W_{o, h}^{\prime}
$$


where $A_{0}^{\prime}, A_{t}^{\prime}$ and $C_{0, h}^{\prime}$ are arbitrary constants and

$$
W_{0}=B_{n}(x, y)
$$

$W_{t}^{\prime}=y^{m b_{t}-m_{m q+m+r}} F_{m p+\bullet}$

$$
\cdot\left(\begin{array}{rrr}
\Delta\left(m ;-n+m-m b_{t}\right), \Delta\left(m ;-n+m-m b_{t}-\left(\beta_{q}\right)+1\right),\left(\left(a_{r}\right)-b_{t}+1\right) & \\
\Delta\left(m ;-n+m-m b_{t}-\left(\alpha_{p}\right)+1\right), & \left(\left(b_{s ;}\right)-b_{t}+1\right) & 2-b_{t}
\end{array} \mid\right.
$$

and

$W_{g, h}^{\prime}=y^{\sigma-m-n-\alpha_{h_{m++m+r}}} F_{m p+s}$

$$
\left(\begin{array}{rr}
\nabla\left(m ; \alpha_{h}+m-g\right), \Delta\left(m ; 1-g-\left(\beta_{q}\right)+\alpha_{h}+m\right),\left(\left(a_{r}\right)+1+\frac{\alpha_{h}+n-g}{m}\right) & ; \frac{\mu(-m)^{m(q-p+1)}}{(\nu x y)^{m}} \\
\Delta\left(m(g) ; 1-g+\alpha_{h}-\left(\alpha_{p ;}\right)+m\right),\left(2+\frac{\alpha_{h}+n-g}{m}\right),\left(\left(b_{s}\right)+1+\frac{\alpha_{h}+n-g}{m}\right)
\end{array}\right) .
$$

Here also the first $s+1$ solutions $W_{0}$ and $W_{t}^{\prime}$ are polynomials, whereas the remaining $m p$ solutions, i.e. $W_{g, h}^{\prime}$, are functions.

VERIFICATION. It can be shown that $W_{t}$ and $W_{g, h}$ satisfy the differential equation (2.4), while $W_{t}^{\prime}$ and $W_{g, h}^{\prime}$ satisfy the equation (2.5). The method of verification is similar to that of Rainville $[2, \S 47]$.

\section{REFERENCES}

1. B. D. Agrawal and I. K. Khanna, Unification of certain classical polynomials, (to appear).

2. E. D. Rainville, Special functions, Macmillan, New York, 1960.

Banaras Hindu University, India 